\title{
Nephrolepis cordifolia: A Review on the fern
}

\author{
F.Johnsy Mary ${ }^{1 *}$, Dr.M.Senthil Kumar ${ }^{2}$, A. Leelavathi ${ }^{3}$, M. Lokesh ${ }^{3}$, M. Mahalakshmi ${ }^{3}$, \\ M. B. Masoor ahmed ${ }^{3}$, A. Meganathan ${ }^{3}$
}

${ }^{1}$ Assistant Professor, ${ }^{2}$ Professor \& Principal, ${ }^{3}$ Annai Veilankanni’s College of Pharmacy, Saidapet, Chennai, Tamil Nadu 600015, India.

Corresponding author: F.Johnsy Mary

Email : sharonangela2011@ gmail.com

Mob: 9677117387

\begin{abstract}
Worldwide, not only is microbiological drug resistance increasing, but so is the burden of liver disease, with approximately 1 million deaths due to liver cirrhosis each year, as well as another 1 million deaths due to viral hepatitis and hepatocellular cancer, according to the World Health Organization (WHO). Many studies have discovered that plant-based phytochemical components such as oleanolic acid, eugenol, and -ionone are effective against a variety of drug-resistant microorganisms, including Streptococcus pneumoniae, Methicillinresistant Staphylococcus aureus (MRSA), and Vibrio parahaemolyticus, among others, and that oleanolic acid and -sitosterol derivatives are In the Himalayan wild fern, Nephrolepis cordifolia, all of these compounds have been shown to be beneficial. A variety of phytochemical compounds found naturally in the Himalayan wild fern Nephrolepis cordifolia (Pani Amla) were investigated in this study, with particular emphasis on their antibacterial, antifungal, anticancer (anti-tumour), and hepatoprotective properties..
\end{abstract}

\section{Introduction}

Ferns like Nephrolepis cordifolia grow in colonies in the wild, with short rhizomes and small scaly tubers at their roots, allowing them to expand swiftly and fiercely. They may thrive in a number of settings, including as an epiphytic and epilithic plant, with fronds that are typically 16-32 inches long and 4 inches wide when mature with a bright green colour [1]. The fern is propagated through spores, stolons, tubers, and rhizomes, and it is also known as erect sword fern, lemon butter fern, ladder fern, and fish bone fern. In Tamil, it is known as moothirakilangu; in Malaysia, it is known as sarawak; and in the hilly region of Sikkim, it is known as pani amla [2]. The Himalayan tribes use this fern as a vegetable and cook it in a number of ways. This fern has historically been used in Indian traditional medicine as a diuretic, a contraceptive, and a treatment for liver issues [3]. This plant's tubers have a high concentration of moisture, fat, glucose, and calcium, while the rhizome section has a high concentration of protein. People in Nepal eat fresh Nephrolepis cordifolia tubers that have been roasted before consuming them. Flowers of the fern Nephrolepis cordifolia are commonly grown as a beautiful plant in various areas of Shillong [4].

\section{Morphological Characters}

The plant is pinnate and can grow up to 3 feet tall and 3 inches broad. Pinnae range in size from 1.5 to 4 inches on either side of the rachis. Each pinnae developing on the rachis is 
oblong or lanceolate in form, with many spore-containing structures called sori, which are generated between the mid-vein of the leaflets and the edges. Rhizomes are covered in brownish scales and have fleshy, egg-shaped tubers. Nephrolepis cordifolia develops round fleshy tubers that are spherical and around $15 \mathrm{~mm}$ in diameter and grow from a network of creeping stalks. Tubers of Nephrolepis cordifolia have high moisture content. This high moisture content in food samples promotes the activity of water soluble enzymes and coenzymes that are beneficial for a variety of metabolic processes [5].

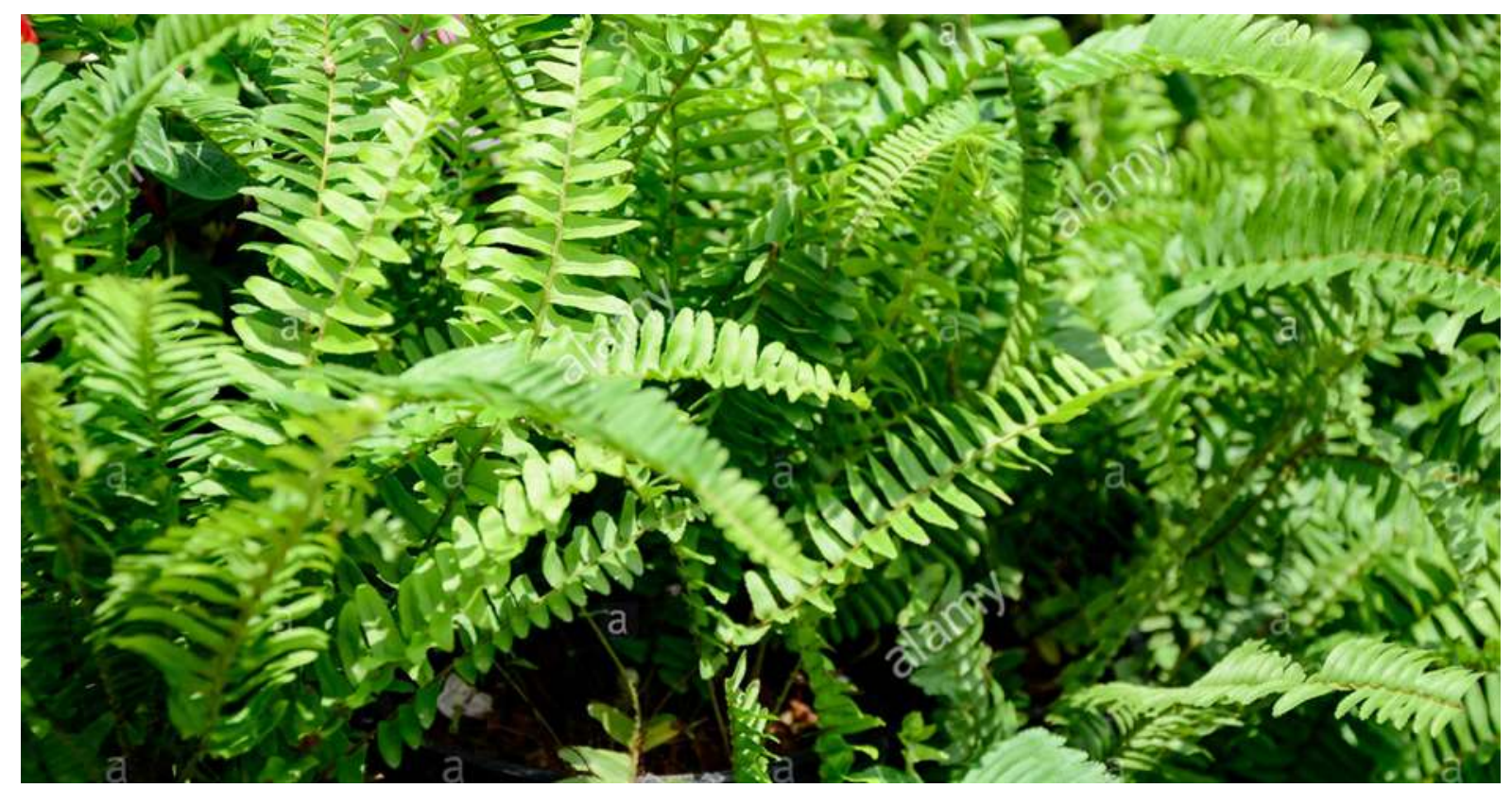

Fig 1: Nephrolepis cordifolia Plant

\section{Phytochemistry}

Epidermal glands cover the many sections of Nephrolepis cordifolia, such as the leaf, rhizome, and rachis. These epidermal glands contain phenolic acids, flavonoids, glycosides, and alkaloids, among other phytochemical compounds. Because of the existence of these compounds, they exhibit antimicrobial action against a variety of microorganisms and are found to be less soluble in water and more soluble in organic solvents such as ethanol, acetone, and methanol. The phytochemical analysis of Nephrolepis cordifolia extract revealed that components such as reducing sugar, tannins, and cardiac glycosides are abundant [6]. Six distinct types of chemicals were isolated using Nephrolepis cordifolia ethanol extract in one experiment. The six isolated and identified compounds are -sitosterol, fern-9(11)-ene, myristic acid, oleanolic acid, triacontanol, and hentriacontanoic acid, all of which are isolated for the first time from this plant. Nonanal (10.6 percent), b-ionone (8.0 percent), eugenol (7.2 percent), and anethol (4.6 percent) were identified in very high concentrations in one investigation on the chemical makeup of essential oil of Nephrolepis cordifolia [7].

\section{Pharmacological activities}

\section{Antibacterial activity}

One research found that plant extract-based oleanolic acid has antibacterial action against vancomycin-resistant enterococci, with a MIC of $8 \mathrm{~g} / \mathrm{ml}$ against vancomycin-resistant enterococci. Oleanolic acid also inhibited the growth of Streptococcus pneumoniae and Methicillin-resistant Staphylococcus aureus (MRSA). Fern-9 (11)-ene was discovered to be sensitive to gramme negative bacteria except E. coli, extremely susceptible to Salmonella 
typhi, and moderately potent against Pseudomona aeruginosa [8]. One experiment study on the antibacterial and antibiofilm activity of eugenol against Vibrio parahaemolyticus demonstrated strong antibiofilm properties of eugenol against environmental and clinical isolates of multidrug resistant bacteria Vibrio parahaemolyticus and 0.1 percent concentration of eugenol is able to decrease biofilms by 3 and $2.5 \mathrm{CFU} / \mathrm{cm} 2$ against both clinical and environmental isolates, respectively. [9]

\section{Antifungal activity}

Several investigations have shown that oleanolic acid has antifungal properties against a variety of harmful fungi. Some studies on oleanolic acid derivatives that have been developed and studied as possible therapeutic agents in the management of microbial illnesses are available. One of the researchers designed and synthesised the 25 oxime ester derivatives of oleanolic acid to study their antifungal activities and discovered that all of the oleanolic acid derivatives showed potential antifungal activity against fungi such as Sclerotinia sclerotiorum and Rhizoctonia solani at concentrations of $50 \mathrm{~g} / \mathrm{ml}$. In one investigation on the impact of eugenol on 31 strains of Candida albicans, it was discovered that eugenol totally suppresses budding and mycelia development and had a MIC of $625 \mathrm{~g} / \mathrm{ml}$ for all of the tested strains of Candida albicans. Another investigation on the impact of the -Ionone molecule on $\mathrm{P}$. expansum discovered that at 0.625 percent concentration of the -Ionone is considerably able to inhibit the development of P. expansum and with no apparent growth of its spores after incubation period [10].

\section{Anticancer activity}

Various in vivo and in vitro investigations have demonstrated anti-tumour and anti-cancer effects of oleanolic acid against tumour and cancer development. Oleanolic acid has been shown to decrease tumour cell development in mice and to reduce the proliferation of hepatocellular cells in the liver. It has been shown that oleanolic acid's anticancer effect is dependent on tumour protein (p53) overexpression, mitochondrial apoptotic pathway activation mediated by cyclooxygenase-2, and cell cycle progression cessation [11]. Another study revealed that when malignant cells from the human bladder are treated with $50 \mathrm{M}$ oleanolic acid, the whole growth process ceases and the shutdown of apoptosis or programme cell death happens owing to blockage of various pathways. In one investigation on malignant melanoma cells, researchers discovered that eugenol serves as a strong antiproliferative agent against malignant melanoma cells when treated with it. A recent invivo investigation on mice revealed that when -sitosterols are fed to mice on a regular basis, the immunological response of the organism is triggered. This study also demonstrates that the cytokines IL-12 and IL-18, as well as natural killer cells, have a significant activity in reducing the frequency of metastases in a lung cancer cell line. Low-level -sitosterol absorption slows cell proliferation in colonic epithelial cells, which further reduces the expression of neoplastic cells during transformation [12].

\section{Hepatoprotective activity}

Oleanolic acid is a pentacyclic terpenoid that is found in plants in the form of free acids and triterpenoid saponin glycosides. Many animal investigations have shown that oleanolic acid plays an essential role in protecting against liver damage caused by CCl4, phalloidin, acetaminophen, and other drugs by lowering blood transaminase levels, therefore preventing 
necrosis of liver cells. Acute hepatic damage of the liver is a potentially fatal illness that can be characterised by caugolopathy. It can be caused by a variety of causes such as viruses, alcohol, and chemicals. Gal N (d-Galactosamine) is a hepatotoxic drug that consumes a high concentration of UTP (uridine triphosphate) in order to block the synthesis of related rna and protein in the liver, causing inflammation and hepatic necrosis. Lps (lipopolysaccharide) stimulates immune cells, which release different inflammatory substances, causing apoptosis and necrosis of hepatic cells in the liver. In one research, it was shown that the -sitosterol derivative sitosterol-N, when administered in different concentrations $(12.5 \mathrm{mg} / \mathrm{kg}$ ) and $(50 \mathrm{mg} / \mathrm{kg})$, aids in therapy and is capable of reducing the necrosis of liver cells caused by lipopolysaccharide and d-Galactosamine [13].

\section{Conclusion}

According to the WHO, many pathogenic microbes are developing resistant to medicines as a result of improper drug usage, posing a significant challenge for researchers. Additionally, the use of synthetic pharmaceuticals is not always a viable option owing to adverse effects on the body. Liver illnesses are becoming a new burden for people all over the world, with about 1 million deaths per year owing to liver cirrhosis and another million deaths due to viral hepatitis and hepatocellular cancer. Some of the components found in Nephrolepis cordifolia include oleanolic acid, eugenol, and -ionone, which have antimicrobial properties against Streptococcus pneumoniae, Methicillin resistant Staphylococcus aureus (MRSA), and Vibrio parahaemolyticus, among others, whereas oleanolic acid and -sitosterol derivatives have been found to be effective against various Not only do the components of Nephrolepis cordifolia have antibacterial and hepatoprotective characteristics, but they are also efficient against various pathogenic fungi and tumour or nercrosis of hepatic cells. Nephrolepis cordifolia is a less-explored wild plant that offers chances for researchers to discover novel phytochemicals concealed within it, but it requires more study effort to determine its phytochemical makeup. All of the naturally occurring compounds in Nephrolepis cordifolia have the potential to be varied into effective medicinal products against multidrug resistant bacteria and hepatocellular necrosis of the liver.

\section{References}

1. Cheng L, Pu BJ, Zhou GY. The effects of physical and chemical factors on differentiation and growth of green globular bodies of Nephrolepis cordifolia. Journal of Tropical and Subtropical Botany, 2001; 9:142-148.

2. Tamang Mahendra, Pal Krishan, Santosh Kumar Rai. Traditional Use of wild plants for food in West Sikkim, India. Int. J of Life Sciences. 2017; 5(4):730-741.

3. Dhiman AK. Ethno medicinal uses of some pteridophytic species in India. Indian Fern Journal. 1998; 15(1, 2):61-64.

4. Chhetri RB. Trend in Ethnodomestication of some wild plants in Meghalaya, North East India. Indian J of Traditional Knowledge. 2006; 53(3):342- 347.

5. Rani D, Khare PB, Dantu PK. In vitro antibacterial and antifungal properties of aqueous and non-aqueous frond extracts of Psilotum nudum, Nephrolepis biserrata and Nephrolepis cordifolia. Indian J Pharmaceut. Sci. 2010; 72:818-822. 
6. Oloyede FA, Ajayi OS, Bolaji IO, Famudehin TT. An Assessment of Biochemical, Phytochemical and Antinutritional Compositions of a Tropical Fern: Nephrolepis cordifolia L. Ife Journal of Science. 2013; 15(3)

7. Liang Zhi-Yuan, Yang Xiao-Sheng, Zhu Hai-Yan, Hao Xiao-Jiang. Chemical constituents of Nephrolepis cordifolia. China National Knowledge Infrastructure. Guihaia, 2008, 3

8. Reddy VL, Ravikanth V, Rao TP, Diwan PV, Venkateswarlu Y. A new triterpenoid from the fern Adiantum lunulatum and evaluation of antibacterial activity. Phytochemistry. 2001; 56(2):173-175. doi:10.1016/s0031-9422(00)00334-4

9. Md Ashrafudoulla, Md Furkanur Rahaman Mizan, Angela Jie-won Ha, Si Hong Park, Sang-Do Ha. Antibacterial and antibiofilm mechanism of eugenol against antibiotic resistance Vibrio parahaemolyticus, Food Microbiology, Volume 91,2020,103-105,

10. Barkai Hassan, Elabed Soumya, Guissi Sanae, Ibn Souda Koraichi Saad. Evaluation of the Antifungal Activities of Three Essential Oil Components against Penicillium expansum Spores. International Journal of Pharmacy and Pharmaceutical Sciences. 2017; 9(8). 56-59

11. Wang X, Bai H, Zhang X, Liu J, Cao P, Liao N, et al. Inhibitory effect of oleanolic acid on hepatocellular carcinoma via ERK-p53-mediated cell cycle arrest and mitochondrialdependent apoptosis. Carcinogenesis 2013; 34:1323-1330.

12. Baskar AA, Al Numair KS, Gabriel Paulraj M, Alsaif MA, Muamar MA et al. $\beta$-sitosterol prevents lipid peroxidation and improves antioxidant status and histoarchitecture in rats with 1,2-dimethylhydrazine-induced colon cancer. J Med Food. 2012; 15:335-343.

13. Yongxia Yin, Xiaofeng Liu, Jinping Liu, Enbo Cai, Hongyan Zhu, Haijun Li et al. Betasitosterol and its derivatives repress lipopolysaccharide/D-galacto samine - induced acute hepatic injury by inhibiting the oxidation and inflammation in mice, Bioorganic \& Medicinal Chemistry Letters. 2018; 28:1525-1533. 